\title{
Galactic astroarchaeology: reconstructing the bulge history by means of the newest data
}

\author{
G. Cescutti ${ }^{1}$ and F. Matteucci ${ }^{1,2}$ \\ 1 Dipartimento di Fisica, Sezione di Astronomia, Universitá di Trieste, via G.B. Tiepolo 11, 34131 Trieste, Italy \\ e-mail: cescutti@oats.inaf.it \\ 2 INAF Osservatorio Astronomico di Trieste, via G.B. Tiepolo 11, 34131 Trieste, Italy
}

Received 31 August 2010 / Accepted 5 October 2010

\section{ABSTRACT}

\begin{abstract}
Context. The chemical abundances measured in stars of the Galactic bulge offer an unique opportunity to test galaxy formation models, as well as to impose strong constraints on the history of star formation and stellar nucleosynthesis.

Aims. The aims of this paper are to compare abundance predictions from a detailed chemical evolution model for the bulge with the newest data. Some of the predictions have already appeared in previous papers $(\mathrm{O}, \mathrm{Mg}, \mathrm{Si}, \mathrm{S}$, and $\mathrm{Ca})$, but some other predictions are new (Ba, $\mathrm{Cr}$, and $\mathrm{Ti})$.

Methods. We computed several chemical evolution models by adopting different initial mass functions for the Galactic bulge and then compared the results to new data including both giants and dwarf stars in the bulge. In this way we can impose strong constraints on the star formation history of the bulge.

Results. We find that to reproduce the metallicity distribution function best, one should assume a flat IMF for the bulge that is not steeper than the Salpeter one. The initial mass function derived for the solar vicinity instead provides a very poor fit to the data. The $[\mathrm{el} / \mathrm{Fe}]$ vs. $[\mathrm{Fe} / \mathrm{H}]$ relations in the bulge are reproduced well by a very intense star formation rate and a flat IMF, as in the case of the stellar metallicity distribution. Our model predicts that the bulge formed very quickly with the majority of stars formed inside the first $0.5 \mathrm{Gyr}$.

Conclusions. Our results strongly suggest that the new data and, in particular, the MDF of the bulge confirm what have been concluded before, that the bulge formed very fast from gas shed by the halo, and that the initial mass function was flatter than in the solar vicinity and in the disk, although not as flat as previously thought. Finally, our model can also reproduce the decrease in the $[\mathrm{O} / \mathrm{Mg}] \mathrm{ratio}$ for $[\mathrm{Mg} / \mathrm{H}]>0$ in the bulge, which is confirmed by the new data and interpreted as the result of mass loss in massive stars.
\end{abstract}

Key words. Galaxy: evolution - Galaxy: bulge - Galaxy: abundances - stars: abundances - nuclear reactions, nucleosynthesis, abundances

\section{Introduction}

The bulges of spiral galaxies are generally distinguished in true bulges, hosted by $\mathrm{S} 0-\mathrm{Sb}$ galaxies and "pseudobulges" hosted in later type galaxies (see Renzini 2006, for references). Generally, the properties (luminosity, colors, line strengths) of true bulges are very similar to those of elliptical galaxies. In the following, we only refer to true bulges and, in particular, to the bulge of the Milky Way. This bulge is, in fact, the best-studied bulge, and several scenarios for its formation have been put forward in past years. As summarized by Wyse \& Gilmore (1992) the proposed scenarios include: i) the bulge being formed by accretion of extant stellar systems that eventually settle in the center of the Galaxy; ii) the bulge being formed by accumulation of gas at the center of the Galaxy and subsequent evolution with either fast or slow star formation; iii) the bulge formed by accumulation of metal enriched gas from the halo or thick disk or thin disk in the Galaxy center. More recently, Elmegreen $(2008,2009)$ has proposed a bulge formation due to coalescence of large clumps in primordial galaxies. These clumps form by gravitational instability. The model requires fast gas assembly and not a hierarchical merging of pre-existing star-rich galaxies as in some hierarchical build-up models. Therefore, this model suggests a fast assembly of bulges.
The metallicity distribution function (MDF) of stars in the bulge and the $[\alpha / \mathrm{Fe}]$ ratios help considerably in selecting the most probable scenario for bulge formation. Matteucci \& Brocato (1990) suggested that, in order to fit the MDF of the bulge, one should assume that it formed very quickly in less than $1 \mathrm{Gyr}$ and, as a consequence of this, they predicted that the $[\alpha / \mathrm{Fe}]$ ratios in the bulge stars should be oversolar for a large interval of $[\mathrm{Fe} / \mathrm{H}]$. Their suggestion was based on the assumption that galaxies of different morphological types suffer different histories of star formation; in particular, ellipticals and true bulges should experience a strong burst of star formation lasting for a short time, whereas spirals and even more irregulars suffer a milder and continuous star formation rate (SFR). These assumptions, coupled with the time-delay between the Fe enrichment from type Ia supernovae (white dwarfs in binary systems) and the $\alpha$-element enrichment from core collapse supernovae (originating in massive stars, type $\mathrm{II}, \mathrm{Ib} / \mathrm{c}$ ) produce a different behavior of the $[\alpha / \mathrm{Fe}]$ vs. $[\mathrm{Fe} / \mathrm{H}]$ relations under different SFRs. In other words, objects such as bulges evolve very fast and the $[\alpha / \mathrm{Fe}]$ ratios in the majority of their stars are expected to be high and oversolar for a large interval of $[\mathrm{Fe} / \mathrm{H}]$. Since star formation is very intense, the bulge very soon reaches a solar metallicity only thanks to the core collapse $\mathrm{SNe}$, which produce some $\mathrm{Fe}$. Then when $\mathrm{SNe}$ Ia, which produce the bulk of Fe, start exploding, the change in the slope occurs at a larger $[\mathrm{Fe} / \mathrm{H}](\sim 0)$ than in the 
solar vicinity ( -1.0). For spiral and irregular instead, the Fe enrichment by core collapse $\mathrm{SNe}$ is much less, due to the milder SFR, and the $[\mathrm{Fe} / \mathrm{H}]$ is still low when type Ia SNe start appearing. Thus the change in slope in irregulars occurs at $[\mathrm{Fe} / \mathrm{H}] \leq-2.0$ (see Matteucci 2001).

Years later, McWilliam \& Rich (1994) observed some $[\alpha / \mathrm{Fe}]$ ratios in stars in Baade's window and concluded that $[\mathrm{Mg} / \mathrm{Fe}]$ was indeed high for a wide range of $[\mathrm{Fe} / \mathrm{H}]$. For other elements, such as oxygen, the situation was not very clear. These data were derived from low-resolution spectra. Minniti (1996) concluded from kinematics and metallicities of red giant stars in the field that the bulge formed quickly by dissipative collapse, from material left over after the formation of the halo. In subsequent years a great deal of observations of bulge stars appeared. Highresolution abundances were derived by Zoccali et al. (2006), Fulbright et al. (2007), Lecureur et al. (2007), and these studies suggest an oversolar and almost constant value for a wide $[\mathrm{Fe} / \mathrm{H}]$ range, even for other $\alpha$-elements such as $\mathrm{O}$, thus confirming that the abundance ratios indicate a fast bulge formation. From the theoretical point of view, Ballero et al. (2007, hereafter BMOR07) present an updated model relative to Matteucci \& Brocato (1990) for the bulge. This model includes stellar feedback and the development of a galactic wind, which occurs when most of star formation in the bulge is over. Very detailed predictions were given in this paper for several elements and for the MDF. The agreement with observations was good, suggesting that the bulge formed on a timescale between 0.3 and $0.5 \mathrm{Gyr}$, that the star formation was much more efficient than in the solar vicinity (by a factor of $\sim 20$ ), and that an IMF that is much flatter than the Scalo (1986) or Kroupa et al. (2001), adopted for the solar neighborhood, was required. In particular, this is required by the observed MDF. Recently, more abundance data for bulge and thick disk giant stars have appeared (Alves-Brito et al. 2010), measuring several $\alpha$-elements and Fe. The observed trends confirm the previous papers and find a similarity between the thick disk and bulge stars. Moreover, Bensby et al. (2010) and Johnson et al. $(2007,2008)$ have for the first time measured the same abundance ratios in microlensed dwarf and subgiant stars in the bulge and find good agreement with the abundances measured in giant stars.

The paper is organized as follows. In Sect. 2 we describe the observational data, in Sect. 3 we briefly describe the chemical model for the bulge, in Sect. 4 we compare our model results with the newest data, and finally in Sect. 5 we summarize our conclusions.

\section{Observational data}

In this work, we choose to use only the most recent observational data for the bulge. We select the chemical abundance data calculated by Bensby et al. (2010), Alves-Brito et al. (2010), and Ryde et al. (2009). The spectra were obtained by the different authors using different techniques: Bensby et al. (2010) perform a detailed elemental abundance analysis of dwarf stars in the Galactic bulge, based on high-resolution spectra that were obtained while the stars were optically magnified during gravitational microlensing events; Alves-Brito et al. (2010) use highresolution optical spectra of 25 bulge giants in Baade's windows and 55 comparison giants (4 halo, 29 thin disk, and 22 thick disk giants) in the solar neighborhood; Ryde et al. (2009) obtain highresolution, near-infrared spectra in the $\mathrm{H}$ band recorded using the CRIRES spectrometer on the Very Large Telescope. The CNO abundances can all be determined from the numerous molecular lines in the wavelength range observed, and the abundances of the $\alpha$ elements $\mathrm{Si}, \mathrm{S}$, and Ti are also determined from the near-IR spectra. For a comparison with our previous work, we decide to show the data selected in Cescutti et al. (2009) for the elements $\mathrm{Mg}$ and $\mathrm{O}$. These data are fully described in McWilliam et al. (2008).

We compared our model results for the MDF in the bulge with the MDF determined by Zoccali et al. (2008). They observed about 800 bulge field $\mathrm{K}$ giants with the GIRAFFE spectrograph of FLAMES at the VLT at spectral resolution $R \sim 20000$. The iron abundances, results of their LTE analysis, allowed an MDF to be constructed for the bulge that, for the first time, is based on high-resolution spectroscopy for each individual star.

\section{The chemical evolution model}

The adopted basic chemical evolution model closely follows the one in BMOR07. The main assumption is that the Galactic bulge formed by the fast collapse of primordial gas (the same gas out of which the halo was formed) accumulating in the centre of our Galaxy. We recall the fundamental ingredients of this model:

- Instantaneous mixing approximation refers to the gas over the whole bulge being homogeneous and well mixed at any time.

- Star formation rate (SFR) parameterized as:

$\psi(r, t)=v G^{k}(r, t)$

where $v$ is the star formation efficiency (i.e. the inverse of the timescale of star formation) in the bulge, $k=1$ is chosen to recover the star formation law employed in models of spheroids (e.g. by Matteucci 1992), and $G(r, t)=$ $\sigma_{\text {gas }}(r, t) / \sigma\left(r, t_{\mathrm{G}}\right)$ is the normalized gas surface mass density (where $\sigma_{\text {gas }}(r, t)$ is the gas surface mass density and $\sigma\left(r, t_{\mathrm{G}}\right)$ is the surface gas density of the bulge at the present time $t_{G}=13.7 \mathrm{Gyr}$ ).

- The initial mass function (IMF) is expressed as a power law with index $x$ :

$\phi(m) \propto m^{-(1+x)}$

within the mass range $0.1-100 M_{\odot}$. In this paper we adopt as a reference model the IMF suggested by BMOR07, namely a two-slope IMF with $x=0.95$ for $M>1 M_{\odot}$ and $x=0.33$ for $M<1 M_{\odot}$, and we also test a one-slope Salpeter (1955) IMF $(x=1.35)$ and the two-slope Scalo (1986) IMF.

- The gas that forms the bulge has a primordial chemical composition, and the accretion rate is given by:

$\dot{G}(r, t)_{\text {inf }}=\frac{A(r)}{\sigma\left(r, t_{\mathrm{G}}\right)} \mathrm{e}^{-t / \tau}$

where $\tau$ is an appropriate collapse timescale, and $A(r)$ is constrained by the requirement of reproducing the current total surface mass density in the Galactic bulge. Actually, we should use the halo chemical composition for the infalling gas, but our simulations have demonstrated that, unless very high $\alpha$-enhancements are adopted, the results are essentially the same.

- The instantaneous recycling approximation is relaxed and stellar lifetimes are taken into account in detail following the prescriptions of Kodama (1997).

- Detailed nucleosynthesis prescriptions are taken from: i) François et al. (2004), who made use of widely adopted stellar yields and compared the results obtained by including these yields in a detailed chemical evolution model 
with the observational data, with the aim of constraining the stellar nucleosynthesis. For low- and intermediatemass $\left(0.8-8 M_{\odot}\right)$ stars, which produce ${ }^{12} \mathrm{C}, \mathrm{N}$, and heavy $s$-elements, yields are taken from the standard model of Van den Hoek \& Groenewegen (1997) as a function of the initial stellar metallicity. Concerning massive stars $(M>$ $10 M_{\odot}$ ), in order to best fit the data in the solar neighborhood, when adopting Woosley \& Weaver (1995) yields, François et al. (2004) find that $\mathrm{O}$ yields should be adopted as a function of the initial metallicity, that $\mathrm{Mg}$ yields should be increased in stars with masses 11-20 $M_{\odot}$ and decreased in stars larger than $20 M_{\odot}$, and that Si yields should be slightly increased in stars above $40 M_{\odot}$. As in BMOR07 we use here their constraints on the stellar nucleosynthesis to test whether the same prescriptions give good results for the Galactic bulge when compared with the newest data; ii) in the range of massive stars we have also adopted the yields of Maeder (1992) and Maeder \& Meynet (1992) containing mass loss. The effect of mass loss is only visible for metallicities $\geq Z_{\odot}$. The use of these yields is particularly important for studying the evolution of $\mathrm{O}$ and $\mathrm{C}$, the two most affected elements (see McWilliam et al. 2008; Cescutti et al. 2009); iii) for Ba, we use the nucleosynthesis prescriptions adopted by Cescutti et al. (2006) to best fit the observational data for this neutron capture element in the solar vicinity. The same nucleosynthesis prescriptions give also good results when applied to dwarf spheroidals (Lanfranchi et al. 2006) and to the Galactic halo using an inhomogeneous model (Cescutti 2008). In particular, we assume that the the s-process fraction of $\mathrm{Ba}$ is produced in low-mass stars $\left(1-3 M_{\odot}\right)$, whereas the r-process fraction of Ba originates in stars in the range 12-30 $M_{\odot}$.

- The type Ia SN rate was computed according to Greggio \& Renzini (1983) and Matteucci \& Recchi (2001). Yields are taken from Iwamoto et al. (1999), which is an updated version of model W7 (single degenerate) from Nomoto et al. (1984). These supernovae are the main contributors of Fe and produce small amounts of light elements; they also contribute to the enrichment in $\mathrm{Si}$ and $\mathrm{Ca}$ to some extent.

- The treatment of the supernova-driven galactic wind is the same as in BMOR07, where we send the reader for details.

\section{Results}

\subsection{Constraints on the IMF}

As in previous papers, we assumed a high efficiency for star formation $\left(v=20 \mathrm{Gyr}^{-1}\right)$ and a short timescale for gas accretion $(\tau=0.3 \mathrm{Gyr})$. It is supposed that the gas that formed the bulge was the gas shed by the halo, so it was slightly enriched in metallicity. The values of the above parameters produce a very fast bulge formation, as required to reproduce the bulge abundance data. We computed several models for the Galactic bulge by varying the IMF: in particular, we adopted i) the very flat IMF as described in BMOR07; ii) the Salpeter (1955) IMF; and iii) the Scalo (1986) IMF. In Fig. 1 we show the predicted $[\alpha / \mathrm{Fe}]$ ratios versus $[\mathrm{Fe} / \mathrm{H}]$ as predicted and observed in the bulge.

This kind of diagram is usually interpreted on the basis of the time-delay between the chemical enrichment from the type II and the type Ia SNe. Another fundamental parameter in this graph is the SFR, which determines the age-abundance relations and the shape of the $[\alpha / \mathrm{Fe}]$ vs. $[\mathrm{Fe} / \mathrm{H}]$ relations (see Matteucci 2001). In particular, the long plateau and the oversolar values observed for the $[\alpha / \mathrm{Fe}]$ ratios in bulge stars extending to solar

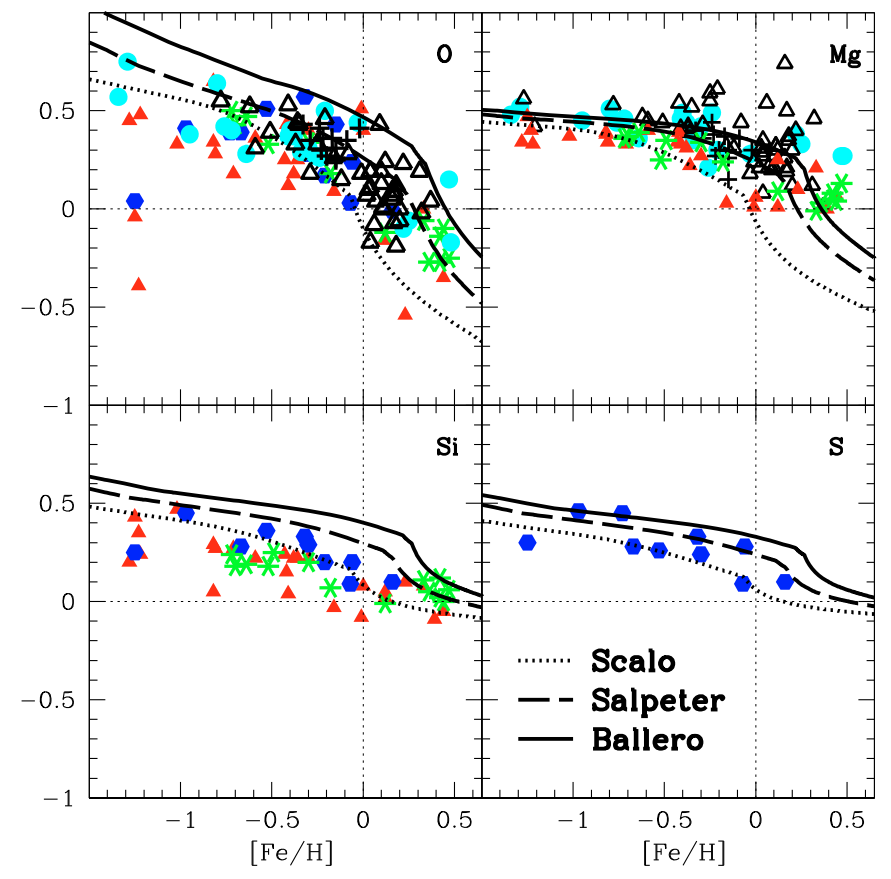

Fig. 1. Comparison between the predictions of our model using 3 different IMF for $[\mathrm{O} / \mathrm{Fe}],[\mathrm{Mg} / \mathrm{Fe}],[\mathrm{Si} / \mathrm{Fe}]$, and $[\mathrm{S} / \mathrm{Fe}]$ vs. $[\mathrm{Fe} / \mathrm{H}]$ and the observations in the bulge. The observational data for the bulge are filled circles from MFR09 and FMR07, the open triangles from Lecureur et al. (2007), the filled triangles from Alves-Brito et al. (2010). The plus signs are the infrared results from Rich \& Origlia (2005), the filled hexagons are the infrared results by Ryde et al. (2009), and the stars are the results for microlensed dwarf stars by Bensby et al. (2010).

metallicity, is reproduced well by a very fast bulge formation. As one can see, different IMFs predict different absolute $[\alpha / \mathrm{Fe}]$ ratios, in the sense that the flatter the IMF, the higher the $[\alpha / \mathrm{Fe}] \mathrm{ra}-$ tios, since there are relatively more massive stars in a flatter IMF than in a steeper one. In addition, different IMFs tend to produce small variations on the knee of the $[\alpha / \mathrm{Fe}]$ ratios, in the sense that a flatter IMF predicts a knee of $[\alpha / \mathrm{Fe}]$ ratio at higher $[\mathrm{Fe} / \mathrm{H}]$ values. Therefore, the length of the plateau can in principle be used to impose constraints on the IMF. However, the knees always occur at $[\mathrm{Fe} / \mathrm{H}]>0$ for the bulge since the strong assumed SFR always induces a very fast increase in the $[\mathrm{Fe} / \mathrm{H}]$, thus having the SNe Ia occurring when the ISM has already reached a solar Fe abundance. It is worth noting that the effect of varying the IMF is different for different element, since it is more evident for $\mathrm{O}$ and almost negligible for $\mathrm{S}$. This of course depends on the specific progenitors of each element and in particular on whether two elements are produced by the same stars or in different mass ranges. The largest difference is, in fact, seen in the $\mathrm{O}$ plot, since $\mathrm{O}$ is mainly produced in massive stars, whereas $\mathrm{Fe}$ is mainly produced in low and intermediate mass stars (SNe Ia).

In the case of $\mathrm{S}$ and $\mathrm{Si}$ instead, $\mathrm{SNe}$ Ia contribute in a nonnegligible way to these two elements. From a look at Fig. 1, we can conclude that the Scalo IMF predicts $[\alpha / \mathrm{Fe}]$ ratios that are too low, whereas the Salpeter and BMOR07 IMFs produce results more in agreement with observations. In Figure 2 we show the predicted and observed $[\mathrm{el} / \mathrm{Fe}]$ vs. $[\mathrm{Fe} / \mathrm{H}]$ for el $=\mathrm{Ba}, \mathrm{Cr}, \mathrm{Ti}$, and $\mathrm{Ca}$. This is the first time that predictions for $\mathrm{Ba}, \mathrm{Cr}$, and $\mathrm{Ti}$ for the bulge are presented. Also in this case the general agreement with the data is good, and the best one is for the BMOR07 and Salpeter IMF, thus reinforcing the conclusion that the IMF in the bulge should be flatter than in the disk, as claimed before 


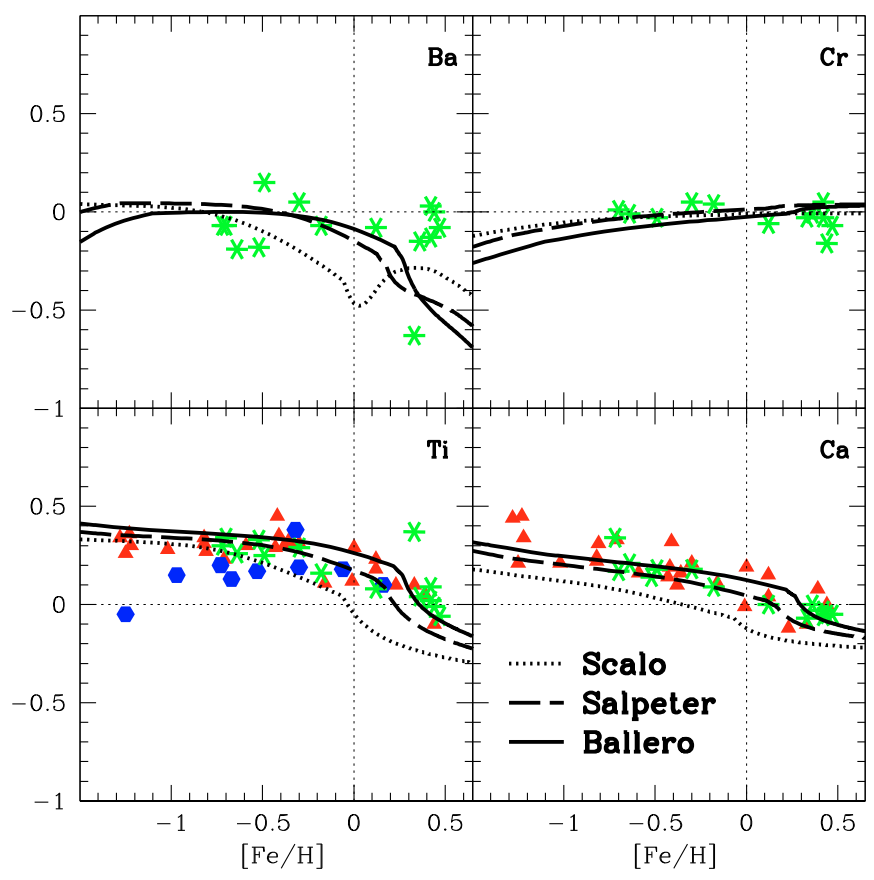

Fig. 2. Comparison between the predictions of our model using 3 different IMF for $[\mathrm{Ba} / \mathrm{Fe}],[\mathrm{Cr} / \mathrm{Fe}],[\mathrm{Ti} / \mathrm{Fe}]$, and $[\mathrm{Ca} / \mathrm{Fe}]$ vs. $[\mathrm{Fe} / \mathrm{H}]$. The symbols for the observational data are the same as in Fig. 1.

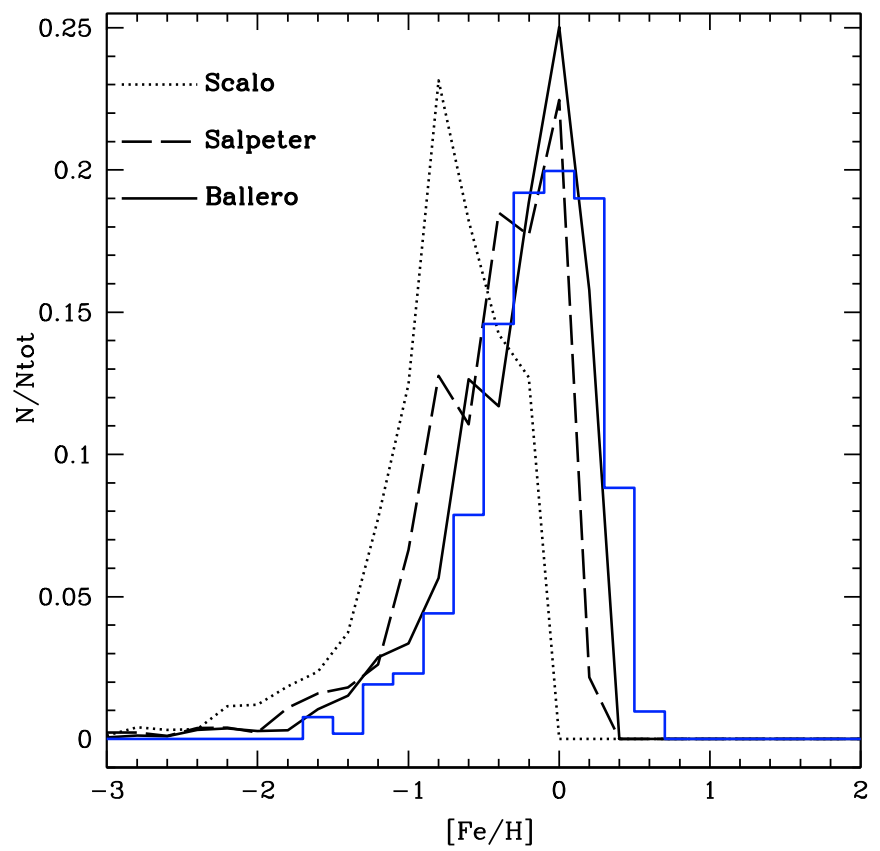

Fig. 3. Comparison between the predictions for the MDF of our model using 3 different IMF and MDF measured by Zoccali et al. (2008) in the bulge stars; the observed MDF is represented by histogram.

in many papers (e.g. Matteucci \& Brocato 1990; Matteucci et al. 1999; BMOR07). This result has important implications for the $[\alpha / \mathrm{Fe}]$ ratios and their behavior with $[\mathrm{Fe} / \mathrm{H}]$ in bulge and thick disk stars (Chiappini et al., in prep.). In fact, there are some indications (e.g. Alves-Brito et al. 2010; Meléndez et al. 2008) that some ratios are the same for bulge and thick disk stars.

However, the most convincing evidence for an IMF flatter in the bulge is provided by Fig. 3 where the predicted and observed stellar metallicity distribution function (MDF) for bulge stars is
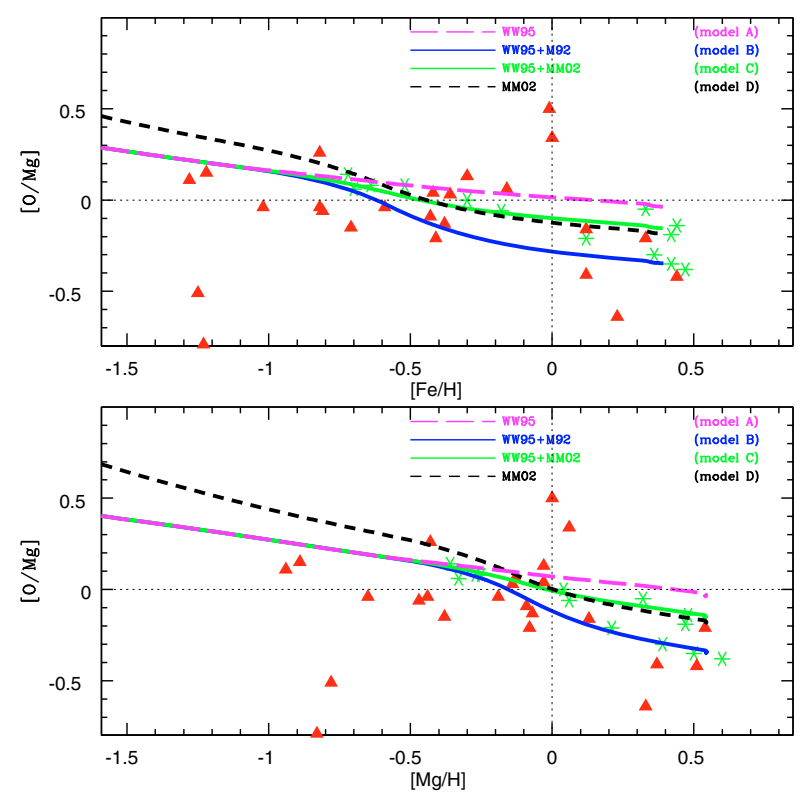

Fig. 4. Comparison between the predictions of our 4 models for $[\mathrm{O} / \mathrm{Mg}]$ vs. $[\mathrm{Mg} / \mathrm{H}]$ (upper panel) and $[\mathrm{O} / \mathrm{Mg}]$ vs. $[\mathrm{Fe} / \mathrm{H}]$ (lower panel). The symbols for the observational data are the same as in Fig. 1.

plotted. As one can see, the position of the peak in the bulge MDF is extremely sensitive to the assumed IMF. A Scalo IMF, which is good for reproducing the solar neighborhood properties, it fails completely for the bulge. The results obtained by Zoccali et al. (2008) are also consistent with the presence of a gradient in the bulge, as are the ones by Minniti (1996). Their findings support our scenario in which both infall and outflow are important and in which the bulge formed very fast by dissipative collapse of gas shed by the halo, rather than the scenario in which the bulge would result solely from the vertical heating of the bar. On the other hand, the presence of a radial metallicity gradient warns us about the limitations of our model, which predicts only a global MDF for the bulge.

\subsection{Constraints on the stellar nucleosynthesis}

A very important result is the change in slope observed for bulge stars in the ratio $[\mathrm{O} / \mathrm{Mg}]$ for $[\mathrm{Mg} / \mathrm{H}]>0$. McWilliam et al. (2008) and Cescutti et al. (2009) interpret this effect as originated from the mass loss in massive stars. In fact, the mass loss only becomes important for metallicities higher than solar and induces the effect of producing more $\mathrm{C}$ and $\mathrm{He}$, which are the elements primarily lost through mass loss by stellar winds, at expense of $\mathrm{O}$, which is produced in a lower amount owing to the loss of its progenitor elements, $\mathrm{C}$ and $\mathrm{He}$. This effect therefore involves only $\mathrm{C}$ and $\mathrm{O}$ among heavy elements, thus producing a lowering of the $[\mathrm{O} / \mathrm{Mg}]$ ratio for $[\mathrm{Mg} / \mathrm{H}]>0$. In this paper we again show the $[\mathrm{O} / \mathrm{Mg}]$ vs. $[\mathrm{Mg} / \mathrm{H}]$ and vs. $[\mathrm{Fe} / \mathrm{H}]$ (Fig. 4 ) compared also with data relative to dwarf stars in the bulge. As one can see, also the dwarf stars confirm the change in slope for the $[\mathrm{O} / \mathrm{Mg}]$ ratio. In this figure the predictions are shown for models with different nucleosynthesis prescriptions (see Cescutti et al. 2009, for details): a model with massive stars evolving without mass loss (model A), which shows no change in the slope, as expected; a model with the mass-loss prescriptions from Maeder (1992) for $Z>Z_{\odot}$ (model B), which gives the best agreement with the observations; two models including the more recent prescriptions for mass loss by Meynet \& Maeder (2002): model 
$\mathrm{C}$ only for $Z>Z_{\odot}$ and model $\mathrm{D}$ for all the metallicities. It is worth noting that the same figures are shown by Alves-Brito et al. (2010), who had the files of our models: however, they plotted $[\mathrm{O} / \mathrm{Mg}]$ vs. $[\mathrm{Fe} / \mathrm{H}]$ twice instead of $[\mathrm{O} / \mathrm{Mg}]$ vs. $[\mathrm{Fe} / \mathrm{H}]$ and $[\mathrm{O} / \mathrm{Mg}]$ vs. $[\mathrm{Mg} / \mathrm{H}]$ and then concluded that the plot as a function of $[\mathrm{Mg} / \mathrm{H}]$ did not provide a good fit. Here we show the correct file and the agreement with the data is indeed very good.

We therefore confirm here that the newest bulge data, including dwarf stars, also indicate a change in slope in the $[\mathrm{O} / \mathrm{Mg}]$ and that this is best explained by mass loss in massive stars, which becomes important for metallicities greater than solar.

\section{Discussion and conclusions}

In this paper we have compared the predictions of a model for the Galactic bulge concerning chemical abundances and the most recent abundance data. The adopted model is that of BMOR07 which assumes a very fast SFR with an efficiency 20 times higher than assumed for the solar vicinity and an infall timescale of $0.3 \mathrm{Gyr}$. It is in fact assumed that the bulge formed by accretion of material lost from the the halo. In particular, some of the predictions $(\mathrm{O}, \mathrm{Mg}, \mathrm{Si}, \mathrm{S}$, and $\mathrm{Ca})$ have already appeared in previous papers, but here they are compared to different and newer data, whereas other predictions concerning $\mathrm{Ba}, \mathrm{Cr}$, and $\mathrm{Ti}$ are new. We tested different IMFs (Salpeter 1955; Scalo 1986; BMOR07). Our main conclusions can be summarized as follows.

- To reproduce the MDF of the bulge stars an IMF that is flatter than the Scalo (1986) one should be assumed. The Scalo IMF, as well as the Kroupa et al. (1993) and Kroupa (2001) IMF which are good for the solar vicinity, do not fit the MDF. This result is very important because it suggests that the IMF in the bulge was different than in the disk. The best IMF is the very flat one suggested by BMOR07. The same conclusion was reached by Ballero et al. (2007b) who analyzed the IMF of Kroupa (2001).

- To best fit the [el/Fe] vs. [Fe/H] relations for $\mathrm{O}, \mathrm{Mg}, \mathrm{Si}, \mathrm{S}, \mathrm{Ti}$, $\mathrm{Cr}$, and $\mathrm{Ba}$, an IMF flatter than the Scalo one is also required. In these cases a Salpeter IMF can also be acceptable. Again, the Scalo and similar IMFs should be rejected. These abundance patterns suggest that the bulge formed very quickly and that the majority of bulge stars were already in place in the first 0.5 Gyr.

- We compared theoretical predictions for the first time with data from dwarf stars in the bulge. These new data agree with the previous ones relative to bulge giants and agree with our predictions. In particular, the dwarf data confirm the change in slope in the $[\mathrm{O} / \mathrm{Mg}]$ vs. $[\mathrm{Mg} / \mathrm{H}]$ observed for $[\mathrm{Mg} / \mathrm{H}]>0$, a trend that has been already successfully interpreted as due to mass loss in massive stars. In particular, a model including stellar yields computed with a high rate of mass loss, such as suggested by Maeder (1992), still gives the best agreement with data.
- We need a flatter IMF than in the solar vicinity, but the novelty over the BMOR07 paper is that we found that a Salpeter IMF with $x=1.35$ over the whole stellar mass range can also reproduce all the bulge data without the need of invoking an extremely flat IMF, as suggested in the above paper, with $x_{1}=0.33$ for $M<1 M_{\odot}$ and $x_{2}=0.95$ for $M>1 M_{\odot}$.

Acknowledgements. We thank Cristina Chiappini and Andy McWilliam for many enlightening discussions. We also thank the referee Dante Minniti for his careful reading and useful suggestions. We acknowledge financial support from PRIN2007-MIUR (Italian Ministry of University and Research) Prot. 2007JJC53X-001.

\section{References}

Alves-Brito, A., Meléndez, J., Asplund, M., Ramírez, I., \& Yong, D. 2010, A\&A, 513, A35

Ballero, S. K., Kroupa, P., \& Matteucci, F. 2007b, A\&A, 467, 117

Ballero, S. K., Matteucci, F., Origlia, L., \& Rich, R. M. 2007a, A\&A, 467, 123

Bensby, T., Feltzing, S., Johnson, J. A., et al. 2010, A\&A, 512, A41

Cescutti, G. 2008, A\&A, 481, 691

Cescutti, G., François, P., Matteucci, F., Cayrel, R., \& Spite, M., 2006, A\&A, 448, 557

Cescutti, G., Matteucci, F., McWilliam, A., \& Chiappini, C. 2009, A\&A, 505, 605

Elmegreen, B. G. 2009, in Galaxy Evolution: Emerging Insights and New Challenges, ed. S. Jogee, L. Hao, G. Blanc, \& I. Marinova, ASP Conf. Ser., 419, 23

Elmegreen, B. G., Bournaud, F., \& Elmegreen, D. M. 2008, ApJ, 688, 67

François, P., Matteucci, F., Cayrel, R., et al. 2004, A\&A, 421, 613

Fulbright, J. P., McWilliam, A., \& Rich, R. M. 2007 ApJ, 661, 1152

Greggio, L., \& Renzini, A. 1983 A\&A, 118, 217

Iwamoto, K., Brachwitz, F., Nomoto, K., et al. 1999, ApJS, 125, 439

Johnson, J. A., Gal-Yam, A., Leonard, D. C., et al., 2007, ApJ, 655, 33

Johnson, J. A., Gaudi, B. S., Sumi, T., et al. 2008, ApJ, 685, 508

Kodama, T. 1997, P.h.D. Thesis, University of Tokio

Kroupa, P. 2001, MNRAS 332, 231

Kroupa, P., Tout, C. A., \& Gilmore, G. 1993, MNRAS, 262, 545

Lanfranchi, G. A., Matteucci, F., \& Cescutti, G. 2006, MNRAS, 365, 477

Lecureur, A., Hill, V., Zoccali, M., et al. 2007, A\&A, 465, 799

Matteucci, F. 1992, ApJ, 397, 32

Matteucci, F. 2001, The Chemical Evolution of the Galaxy (Dordrecht, Kluwer), ASSL, 253, 293

Matteucci, F., \& Brocato, E. 1990, ApJ, 365, 539

Matteucci, F., \& Recchi, S. 2001, ApJ, 558, 351

Matteucci, F., Romano, D., \& Molaro, P. 1999, A\&A, 341, 458

Maeder, A. 1992, A\&A, 264, 105

McWilliam, A., \& Rich, R. M. 1994, ApJS, 91, 749

McWilliam, A., Matteucci, F., Ballero, S. K., et al. 2008, AJ, 136, 367

Meléndez, J., Asplund, M., Alves-Brito, A., et al. 2008, A\&A, 484, L21

Meynet, G., \& Maeder, A. 2002, A\&A, 390, 561

Minniti, D. 1996 ApJ, 459, 175

Nomoto, K., Thielemann, F.-K., \& Wheeler, J. C. 1984, ApJ, 279, 23

Renzini, A. 2006, ARA\&A, 44, 141

Rich, R. M., \& Origlia, L. 2005, ApJ, 634, 1293

Ryde, N., Edvardsson, B., Gustafsson, B., et al. 2009, A\&A, 496, 701

Salpeter, E. E. 1955, ApJ, 121, 161

Scalo, J. M. 1986, FCPh, 11, 1

van den Hoek, L. B., \& Groenewegen, M. A. T. 1997, A\&AS, 123, 305

Woosley, S. E., \& Weaver, T. A. 1995, ApJ, 101, 181

Wyse, R. F. G., \& Gilmore, G. 1992, AJ, 104, 144

Zoccali, M., Lecureur, A., Barbuy, B., et al. 2006, A\&A, 457, 1

Zoccali, M., Hill, V., Lecureur, A., et al. 2008, A\&A, 486, 177 